\title{
Optimizing resiliency of reserve networks to climate change: multispecies conservation planning in the Pacific Northwest, USA
}

\author{
CARLOS CARROLL*, JEFFREYR. DUNK†† and ATTE MOILANEN§ \\ *Klamath Center for Conservation Research, PO Box 104, Orleans, CA 95556, USA, †Department of Environmental and Natural \\ Resource Sciences, Humboldt State University, Arcata, CA 95521, USA, †Redwood Sciences Laboratory, Pacific Southwest Research \\ Station, USDA Forest Service, 1700 Bayview Drive, Arcata, CA 95521, USA, §Department of Biological and Environmental \\ Sciences, PO Box 65, FI-00014, University of Helsinki, Helsinki, Finland
}

\begin{abstract}
The effectiveness of a system of reserves may be compromised under climate change as species' habitat shifts to nonreserved areas, a problem that may be compounded when well-studied vertebrate species are used as conservation umbrellas for other taxa. The Northwest Forest Plan was among the first efforts to integrate conservation of wideranging focal species and localized endemics into regional conservation planning. We evaluated how effectively the plan's focal species, the Northern Spotted Owl, acts as an umbrella for localized species under current and projected future climates and how the regional system of reserves can be made more resilient to climate change. We used the program MAXENT to develop distribution models integrating climate data with vegetation variables for the owl and $\mathbf{1 3 0}$ localized species. We used the program zONATION to identify a system of areas that efficiently captures habitat for both the owl and localized species and prioritizes refugial areas of climatic and topographic heterogeneity where current and future habitat for dispersal-limited species is in proximity. We projected future species' distributions based on an ensemble of contrasting climate models, and incorporating uncertainty between alternate climate projections into the prioritization process. Reserve solutions based on the owl overlap areas of high localized-species richness but poorly capture core areas of localized species' distribution. Congruence between priority areas across taxa increases when refugial areas are prioritized. Although corearea selection strategies can potentially increase the conservation value and resilience of regional reserve systems, they accentuate contrasts in priority areas between species and over time and should be combined with a broadened taxonomic scope and increased attention to potential effects of climate change. Our results suggest that systems of fixed reserves designed for resilience can increase the likelihood of retaining the biological diversity of forest ecosystems under climate change.
\end{abstract}

Keywords: bioclimatic niche, connectivity, conservation prioritization, global change, spatial optimization, species distribution modeling, spotted owl, Zonation

Received 9 March 2009 and accepted 25 April 2009

Introduction

Regional conservation plans often have limited taxonomic breadth due to the lack of distributional data on the majority of species inhabiting an ecosystem. This increases the challenges to evaluating the effectiveness of such reserves under climate change as species' habi-

Correspondence: Carlos Carroll, e-mail: carlos@klamathconservat ion.org tat migrates to nonreserved areas (Araújo et al., 2004). Recent efforts have combined new robust habitat modeling methods for presence-only data (MAXENT; Phillips et al., 2006; Phillips \& Dudik, 2008) with reserve selection software adapted to process the large numbers of resultant habitat suitability surfaces (ZONATION; Moilanen et al., 2005; Moilanen \& Kujala, 2008) to identify reserve systems that optimize habitat quality and connectivity for many species across large regions 
(Kremen et al., 2008; Leathwick et al., 2008; Thomson et al., 2009). Here we extend this approach to design reserve networks that are resilient to climate change and balance goals for wide-ranging focal species and a broad suite of localized endemics. We projected future distributions based on an ensemble of contrasting general circulation models (GCM) (Araújo \& New, 2007), and incorporated uncertainty between alternate climate projections into the prioritization process (Moilanen et al., 2006). We applied these methods to assess and suggest revisions to a regional conservation plan in the Pacific Northwest, USA.

\section{The Northwest Forest Plan (NWFP)}

The US federal NWFP, initiated in 1994, was among the first efforts globally to integrate multiple conservation goals into forest planning at broad spatial scales (USDA \& USDI, 1994; Thomas et al., 2006). The Northern Spotted Owl (Strix occidentalis caurina, henceforth 'the owl') was listed in 1990 as a threatened species under the US Endangered Species Act due to declining population trends related to the loss of older coniferous forest habitat to timber harvest (Noon \& Blakesley, 2006). The NWFP sought to ensure viable populations of the owl and other old-growth-associated species on US federal lands by coordinating regional habitat management across multiple federal ownerships encompassing the range of the owl within the northwestern United States (USDA \& USDI, 1994; Noon \& Blakesley, 2006).

Focal or umbrella species are often used in conservation planning to complement efforts to capture rarespecies locations and vegetative communities (Noss \& Cooperrider, 1994), but have been challenged as performing little better at capturing habitat for a broad suite of species than would be expected by chance (Andelman \& Fagan, 2000). Although the owl has served as a de facto 'umbrella' species for conserving the community of species associated with older forests in the US Pacific Northwest, its effectiveness in this role has not been tested throughout the NWFP region. Here we assess the adequacy of the NWFP land allocations for the owl and localized species, the ability of the owl to serve as a coarse-filter umbrella species for the suite of localized species, and how these conclusions change when climate change is considered.

Before the implementation of the NWFP, forested federal lands in this $\sim 230000 \mathrm{~km}^{2}$ region were largely divided between congressional reserves (e.g., parks and wilderness areas) and nonreserved lands generally open to timber harvest. The NWFP subsequently created an additional management category, late-successional reserves (LSR), of similar total area as congressional reserves, where only limited timber har- vest was allowed (USDA \& USDI, 1994). For our analysis, we divided the NWFP region into four general land management categories: congressional reserves, LSR, matrix, and private lands, which cover $14.2 \%$, $13.9 \%, 23.2 \%$, and $48.8 \%$ of the analysis area, respectively. The matrix category used here is broader than the NWFP's matrix designation, also encompassing administratively withdrawn areas $(2.7 \%)$ and management categories that have limited restrictions on timber harvest, and public lands administered by states and federal agencies not subject to NWFP mandates. The matrix category also includes the NWFP's riparian reserves, stream-side buffer zones with restrictions on logging that have not been mapped at regional scales. Thus our analysis is somewhat conservative in estimating the spatial extent of the current reserve network.

The NWFP combined a network of large reserves designed around the needs of a wide-ranging focal species, the owl, with a fine-scale strategy to survey for and manage known sites for a large suite of localized (rare and endemic) species (Molina et al., 2006; Raphael \& Molina, 2007). An expert-based screening process was used to identify over 400 species that were suspected of being associated with the region's old-forest ecosystems but either too little known to ensure that the new LSR system was adequate to provide for their persistence, or too localized in distribution to be adequately conserved by a conservation plan based on large reserves designed to enhance owl persistence (Molina et al., 2006; Raphael \& Molina, 2007). Because the vast majority of these species were rare and endemic, we refer to this group as 'localized' species, to contrast with the widely distributed owl. The NWFP mandated that distributional data would be collected for these 'Survey and Manage' species before management activities (e.g., timber harvest) occurred on federal lands. Despite the limitations inherent in such nonsystematic sampling, this represented an unprecedented opportunity to enhance understanding of a broad suite of old-forest associated taxa (Olson et al., 2007; Raphael \& Molina, 2007). Because the NWFP's fine-scale component proved to be much more onerous and expensive than anticipated, a rigorous assessment of the conservation needs of localized species and their overlap with those of the owl would greatly increase the efficacy of the NWFP approach (Molina et al., 2006; Raphael \& Molina, 2007).

In this study, we use robust modeling techniques suitable for modeling of species distribution using presence-only datasets such as produced by the NWFP's nonsystematic surveys. We identify reserve networks that optimize habitat representation for a large suite of species using the software ZONATION (Moilanen et al., 2005). We demonstrate new methods of addressing 
uncertainty between climate projections, integrating climate data with other habitat variables, and prioritizing areas based on proximity of habitat under current and changed future climates. Our results have wide relevance for other regional conservation planning efforts that seek to integrate the conservation needs of wide-ranging and localized species under climate change, as well as offering general insights into the efficacy of fixed reserves and contrasts between taxa in response to climate.

\section{Materials and methods}

\section{Species distribution and environmental data}

The study area was based on the extent of the NWFP, which stretches from the Cascade Range to the Pacific Ocean in Washington and Oregon, and covers portions of northwestern California (Thomas et al., 2006) (Fig. 1). The study region covered $83.9 \%$ of the NWFP region's public lands but, due to limitations in the spatial extent of environmental variables, excludes the extreme southern portions of the NWFP area, an area of predominantly private lands which are not subject to the NWFP's management mandates. Ecologists typically divide the NWFP region into ecologically coherent subregions termed ecoprovinces (Fig. 1). The Willamette Valley and Washington Lowlands ecoprovinces were within the extent of analysis but not summarized in the ecoprovince-level results due to their minimal extent of old forest habitat and public lands.

Survey data for localized species consisted of 31100 records on 278 species (Table S1), dating primarily (>99\%) from 1993 to 2003, derived from the Interagency Species Management System (ISMS) database of the US Forest Service Pacific Northwest region (Molina et al., 2006). The Survey and Manage program assigned species to categories that mandated either preproject surveys (e.g., for mollusks) or extensive surveys for groups such as fungi for which predisturbance surveys were not practical (Molina et al., 2006). To create spotted owl distribution models, we used a dataset from Carroll \& Johnson (2008) containing locations of owl nest sites or activity centers derived from digital databases of surveys of owl occupancy and reproductive status from the late 1980s through 2000 for Oregon and Washington and primarily (93\%) from 1987 to 2006 for California. Spatially biased survey effort typical of found data presents a major challenge to distribution modeling (Phillips et al., 2009). To reduce this problem, we thinned data to achieve a minimum separation for each species of $1 \mathrm{~km}$ between locations, using a geographic information system routine that identified clusters of adjacent records and then reduced the set of such

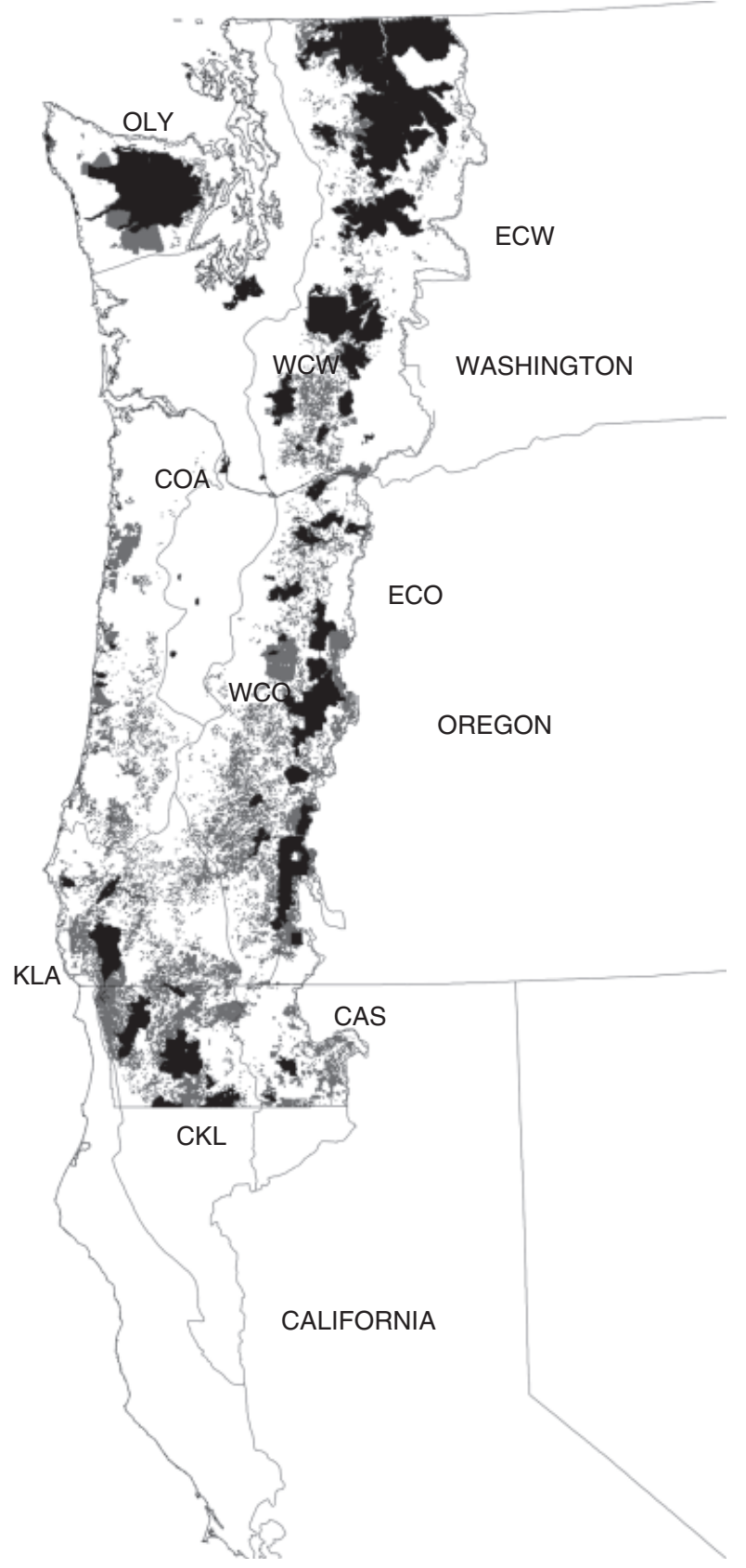

Fig. 1 Map of planning region with ecoprovinces. Ecoprovinces are as follows: OLY, Olympic Mountains; WCW, Western Washington Cascades; ECW, Eastern Washington Cascades; COA, Oregon Coast; WCO, Western Oregon Cascades; ECO, Eastern Oregon Cascades; KLA, Oregon Klamath; CKL, California Klamath, and CAS, California Cascades. The Willamette Valley and Washington lowlands ecoprovinces were within the extent of analysis but not summarized in the ecoprovince-level results due to their minimal extent of old forest habitat and public lands. Priority areas identified in a constrained solution for scenario 3 (interacting current and near-future habitat; see text) are shown in grey with congressional reserves shown in black. 
records to one record randomly selected from that set. A $1 \mathrm{~km}$ separation threshold made it unlikely that multiple records would be included from the same 'site,' e.g., timber sale unit, assessed in preproject surveys. We then limited analysis to the owl and the 130 localized species with 10 or more records remaining after thinning (Fig. 2) (Kremen et al., 2008; Wisz et al., 2008). The 130 localized species comprised 75 fungi, 21 lichen, 10 bryophytes, eight vascular plants, 12 mollusks, two amphibians, a mammal, and a bird (Table S1).

We used data on conifer forest age class developed for the NWFP region through classification of Landsat Thematic Mapper satellite imagery (Strittholt et al., 2006). The vegetation data was aggregated from its original $30 \mathrm{~m}$ resolution to 1 ha resolution for use in modeling. Our predictor variables were the proportion of old conifer forest ( $>150$ years) and mature conifer forest (50-150 years) at two spatial scales, 1 ha and within a $1 \mathrm{~km}^{2}$ area centered on each 1 ha cell. We represented 'current' climate with a model-derived dataset of temperature and precipitation for the conterminous United States and portions of Canada and Mexico (Maurer et al., 2002). The data are closely calibrated to observed climate data (e.g., point observations) but are modeled to maximize comparability of spatial and temporal resolution with those of GCM simulations of future climate scenarios (Maurer et al., 2002). We maintained compatibility with previous ana-

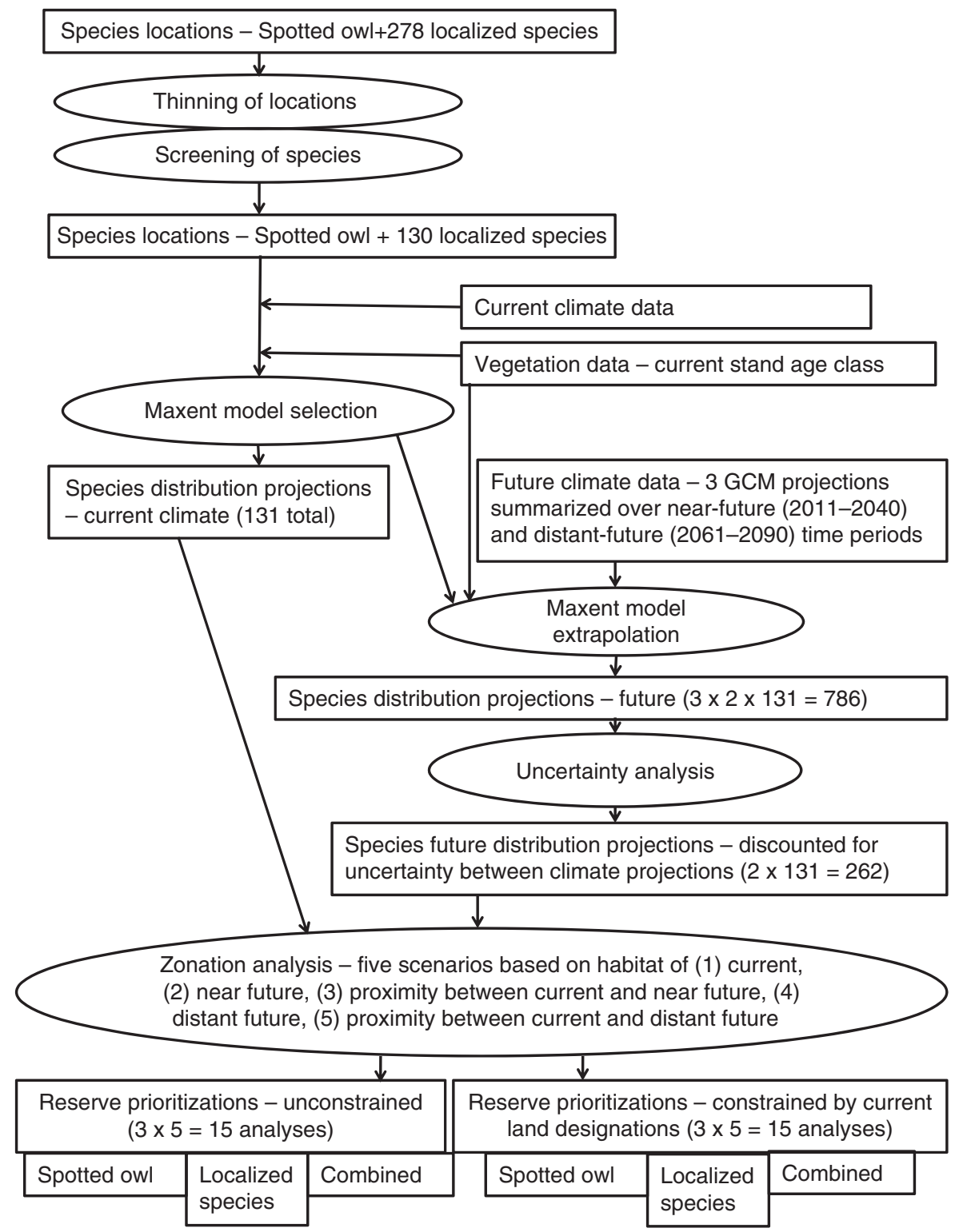

Fig. 2 Flowchart of stages of analysis and data inputs used in multispecies reserve selection for the Pacific Northwest, USA. 
lyses (e.g., Lawler et al., 2006, 2009) by using the period $1961-1990$ as a standard timeframe to represent 'current' climate.

To analyze potential 'future' climate projections, we followed Salathé (2005) in focusing on three representative simulations selected from a group of nine simulations that contrasted in both the GCM and Intergovernmental panel on Climate Change (IPCC) emissions storyline (socioeconomic and population trajectory) used. The three simulations were typified as a lowest warming (GCM GISS_ER with IPCC storyline B1), moderate warming (GCM ECHAM5 SRES with storyline A2), and highest warming scenario (GCM IPSL_CM4 with storyline A2) (Salathé, 2005). Recent observed trends in emissions resemble or exceed those of the A2 IPCC storyline, which represents a future with more rapid economic growth and rapidly rising emissions than are seen in the B1 storyline (Rahmstorf et al., 2007). The data were produced by statistical downscaling the GCM output to a resolution of $1 / 8$ degree (approximately $10 \mathrm{~km} \times 14 \mathrm{~km}$; Salathé, 2005). We resampled both current and future climate data to 1 ha resolution for our habitat modeling. We selected two time periods for analysis: near future (2011-2040) and distant future (2061-2090). We derived the following climate variables from current and projected-future monthly means: mean annual and mean annual standard deviation of temperature and precipitation, as well as mean values for temperature and precipitation from January (wettest, coldest month), 'spring' (March, April, May), and July (driest, hottest month). Because no dynamic vegetation models have yet been developed that predict forest age class based on changing climate at the spatial resolution and regional extent considered here, the old and mature forest vegetation classes were assumed to remain static during the 50-100 year timeframe of our projections.

\section{Environmental niche modeling using MAXENT}

We used MAXENT (Phillips et al., 2006; Phillips \& Dudik, 2008) to evaluate and model relationships between the climate and vegetation variables and the occurrence of the 130 localized species and the owl. MAXENT was designed for use with presence-only data, and performed well in comparison with 15 alternate methods on a wide variety of taxa in diverse regions (Elith $\mathrm{et}$ al., 2006). It has shown especially robust performance in comparison to alternate methods at small sample sizes (e.g., 10 records as here; Hernandez et al., 2006; Pearson et al., 2007; Wisz et al., 2008). MAXENT uses a 'maximum entropy' approach that compares presence locations to a random subset of $\sim 10000$ background or available locations (Phillips et al., 2006). The MAXENT software facilitates projection of a model developed in one study area or time period onto new areas (e.g., to project invasive species risk) or time periods, as here.

Because so little was known about the vast majority of species we evaluated, developing species-specific $a$ priori models from the literature was not feasible. We developed a set of 30 climate models and 10 vegetation models which were then applied to each species (Table S2). We ranked models using the mean rank of AUC and gain and combined the top-ranking climate and vegetation models. AUC evaluates a model's discriminatory ability, whereas gain uses the average log probability of presence samples to evaluate how different, on average, presence locations are to background locations (Phillips et al., 2006). The highest ranking model among the combination, climate-only, or vegetationonly models was then used to predict distribution throughout the study area under current climate conditions. We extrapolated the best current models using MAXENT to predict distribution given projected temperature and precipitation for the three future climate simulations during two future time periods (20112040, 2061-2090) $(131 \times 3 \times 2=786$ prediction maps $)$. We followed the framework of info-gap decision theory (Ben-Haim, 2006) in discounting future occurrence values to account for uncertainty between the three different climate simulations. We derived discounted occurrence values at a $1 \mathrm{~km}$ resolution by subtracting one-half the standard deviation between occurrence values under the three simulations from the mean of occurrence values under those simulations (Moilanen $e t$ al., 2006).

\section{Landscape prioritization using ZONATION}

We identified priority areas using the ZONATION method and software (Moilanen et al., 2005, 2009). ZONATION produces a hierarchical ranking of conservation priority over the entire landscape, accounting for factors such as locally varying habitat quality for each species and land cost. We primarily used the core-area analysis variant, which emphasizes solutions that include high-quality locations for all species, while accounting for priorities (weights) given to them. The core-area variant of ZONATION minimizes loss of conservation value based on local occurrence levels of species, the weights given for species, the amount of the species distribution remaining, past distributional loss and land cost. Complementarity of species composition is also inherent in the formulation. Lowest ranks are given for grid cells that do not contain a valuable occurrence for any species. Highest ranked cells include the highest densities (local occurrence levels) of the species with highest weights. The mathematical details and principles of 
core-area ZONATION are described in Moilanen et al. (2005) and Moilanen (2007), and in the ZONATION user manual (Moilanen \& Kujala, 2008); illustrative regionalscale examples of the use of core-area ZONATION include Leathwick et al. (2008) and Kremen et al. (2008).

In addition to core-area analysis, two alternative prioritization rules, additive-benefit and traditional target-based prioritization, are available in ZONATION (Moilanen, 2007). The additive benefit function bases selection on a cell's weighted summed occurrence value over all species, thus changing the balance of selection to favor species-rich areas over areas with a high occurrence value for one or a few species. In order to evaluate the effects of prioritization method, we also derived additive-benefit solutions for a subset of the scenarios (unconstrained, noninteracting scenarios as described below). Although our habitat modeling had incorporated fine-scale (1 ha) environmental data relevant to localized species, we aggregated MAXENT output to a $1 \mathrm{~km}$ resolution for use in ZONATION. This produced landscape prioritizations at a scale relevant to regional planning. Because ZONATION output typically serves as one of several sources of information for decisions concerning zoning of landscapes for conservation management, we refer to the network of priority areas identified by ZONATION as solutions rather than as reserve networks.

We identified solutions that were resilient to climate change by using the distribution interactions component of ZONATION (Moilanen \& Kujala, 2008; Rayfield et al., 2009). This component transforms the distribution of one conservation target based on its proximity to the distribution of another conservation target. These two distributions could be those of a consumer and its resource (Rayfield et al., 2009), or, as here, the current and future distribution of a species. The distribution interaction transform uses a species-specific dispersal kernel to set the relevant scale of connectivity. The connectivity distribution receives high values at highquality locations where both distributions overlap. If the two distributions are spatially distinct, then connectivity is highest around the closest edges of the distributions. Mathematically, transformation of feature (e.g., species) $j$ by distribution of feature $k$ is of type $p_{i j}{ }^{\prime}=p_{i j} \times C_{i k}$, where $p_{i j}{ }^{\prime}$ is the transformed value of feature $j$ in cell $i, p_{i j}$ is the original value and $C_{i k}$ is the connectivity of location $i$ to the distribution of feature $k$. All locations $i$ are transformed, and $C_{i k}$ involves a distance-scaled summation over all elements of the distribution of feature $k$ (Moilanen \& Kujala, 2008).

By prioritizing areas based on connectivity between current and future habitat, it is possible to identify priority areas that are not only currently valuable for the species but which also facilitate dispersal to ex- pected future distribution areas. We parameterized the interactions between current and future time periods to correspond to a negative exponential distribution giving a mean dispersal distance of $10 \mathrm{~km}$ over the entire 50 or 100-year period. Because scarcity of biological data on the majority of the localized species (Table S1) prevented estimation of species-specific dispersal distances, we chose $10 \mathrm{~km}$ as a conservative estimate of potential dispersal over a multidecadal period. Although the dispersal kernel was based on Euclidean distance, such distances only approximates dispersal probability on real landscapes due to the existence of features such as barriers. We developed scenarios that explored interactions between current and near future (2011-2040) climate. We compared those to areas identified by scenarios incorporating interactions between current and distant future (2061-2090) climate.

Five scenario groups were analyzed that sought to optimize the reserve network respectively for (1) current habitat; (2) near-future habitat; (3) interacting current and near-future habitat; (4) distant future habitat; and (5) interacting current and distant-future habitat. Each of the five scenario groups contained three alternate scenarios with three contrasting sets of conservation goals: localized species, focal species (owl), and combined localized and focal species (15 scenarios in all; Fig. 2). In scenarios with combined goals, in order to balance focal vs. localized species, a higher weight of 10 was given to the single focal species than the weight of one given to each of the 130 localized species.

For each of the 15 scenario-goal combinations described above, we developed unconstrained solutions that built reserve networks from habitat throughout the NWFP region, as well as constrained solutions in which the location of priority areas was constrained to certain management categories (for 30 analyses in total; Fig. 2). The constrained solutions retained current congressional reserves and allocated additional reserves equal in extent to the current LSR network (13.9\% of analysis area) to any location within the current LSR or matrix land management categories. Whereas unconstrained solutions were informative as to general trends in species distribution, constrained solutions allowed assessment of how public-lands management might adapt to climate change given more limited existing options for reserve placement.

\section{Characterization and evaluation of effectiveness of solutions}

For unconstrained solutions, we characterized largescale trends in geographic location of priority areas by measuring the proportion of each ecoprovince falling within priority areas under each scenario. We assessed 
the proportion of the area of each unconstrained solution falling within the four management categories, as compared with the proportion expected based on the total area of land in that management category, in order to assess current and future efficacy of the current reserve system. For constrained solutions, we assessed the proportion of each ecoprovince's LSR and matrix management categories falling within priority areas under each scenario. We assessed the absolute proportion of the area of each constrained solution falling within these two management categories and compared this proportion with the proportion expected based on area in that management category.

We evaluated the effectiveness of the owl as a focal species in capturing habitat for localized species, and whether that role was enhanced or compromised by climate change, by measuring the cross-correlation coefficient (Goodchild, 1986) between priority rankings by cell for owl-based and localized-species based solutions. We also compared the degree to which constrained owl-based solutions captured areas within a localized-species based constrained solution. We evaluated the effectiveness of unconstrained solutions at capturing habitat for the owl and localized species by assessing over all species the mean and minimum proportion of habitat within the solution as compared with the regional habitat total. Mean and minimum are both relevant metrics in that a reserve network that performs relatively well for all species may be preferred to one that performs better on average but at the price of capturing very little habitat for certain species. We assessed the mean proportion of a particular solution required to capture $90 \%$ of the habitat value of a species contained within the entire extent of that solution. This allowed assessment of the degree to which a solution captures core areas of the species distribution vs. areas where many species occur at moderate levels (Moilanen \& Kujala, 2008).

\section{Results}

\section{MAXENT models of species distribution}

A climate model with temperature and precipitation from January and July was the top-ranking climate model for 57 of the 130 localized species and the owl, and second ranking for 28 other species. A climate model with mean and standard deviation of temperature and precipitation was the top-ranking climate model for 30 species and second ranking for another 23 species. The vegetation model with all vegetation variables was the top-ranking vegetation model for 109 of the localized species and for the owl. A model combining the best vegetation and best climate model outperformed both the best climate and best vegetation model for 126 of 130 localized species and the owl. The best model for the owl showed an AUC of 0.849, falling within the 'useful' model category (AUC of 0.7-0.9; Swets 1988). Mean AUC of best models across 130 localized species was 0.929 , indicating 'highly accurate' models (Swets 1988). However, high AUC values for localized species models may be due in part to their restricted range in comparison to the extent of the analysis area (VanDerWal et al., 2009).

\section{Performance of unconstrained solutions}

Trends over the five scenario groups in the proportion of each ecoprovince within ZONATION priority areas for localized species varied according to six distinct patterns (Table S3, Fig. S1a, Fig. S2). These trends appear to be driven by three major factors; latitude, elevation, and topographic and climatic heterogeneity. Contrasts between ecoprovinces in their latitudinal position resulted in declines in ZONATION priority areas in southern ecoprovinces, increases in northern provinces, and stability in ecoprovinces at intermediate latitude. Southern provinces with high elevation areas [CAS (see Fig. 1 legend for ecoprovince codes)] showed intermediate resilience (stable or increasing proportion of area within solution until 2040, declining afterwards). Ecoprovinces with high topographic and climatic heterogeneity (KLA, OLY) retained their importance (proportion of province within priority areas) under climate change, especially in the two scenarios in which dispersal was considered (scenarios 3 and 5, which prioritized proximity between current and near-future and distantfuture habitat, respectively). Correlations between localized-species-based solutions over climate timesteps were lower for a core-area selection strategy that prioritized high-quality habitat than for an additivebenefit selection strategy that prioritized areas of moderate quality for many species $(0.682$ vs. 0.939 for current to near-future, and 0.568 vs. 0.865 for current to distant-future, respectively).

The proportion of each ecoprovince within ZONATION priority areas for the owl was generally more stable under climate change than it was for localized-species based solutions (Fig. S1a). For the owl, results from scenarios where dispersal was considered ( 3 and 5 ) were more similar to those without dispersal ( 2 and 4$)$ than was the case for localized species. Unconstrained solutions for the owl (Fig. 2) under current climate placed $62.4 \%$ of priority areas within LSR or matrix, whereas solutions for localized species had comparatively more focus on private lands, as well as increased focus on congressional reserves (Fig. 3). LSR formed approximately twice the proportion of the ZONATION 


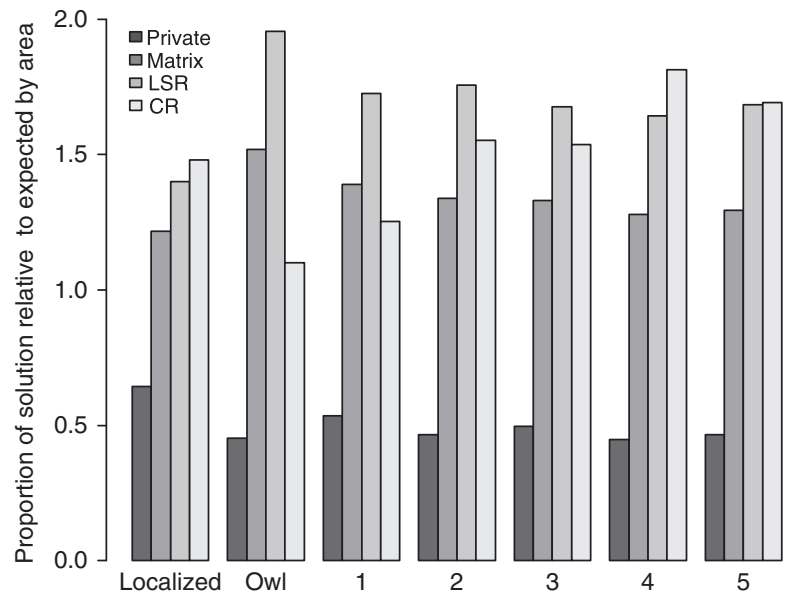

Fig. 3 Relative contribution of management categories to the ZONATION solution under unconstrained scenarios, as compared with that expected by the area of each category. Scenarios 1 through 5 are as above with composite goals. 'Localized' and 'owl' scenarios represent scenario 1 (current habitat) but with habitat goals for only localized species or the owl, respectively. LSR, late-successional reserve; CR, congressional reserve.

solution for the owl than would be expected based on their area alone (Fig. 3). For composite solutions under climate change, the role of congressional reserves increased whereas that of all other management categories decreased slightly. When compared with their expected role based on area (Fig. 3), private land, matrix, and LSR showed a lower than expected, greater than expected, and much greater than expected contribution to the solution under current and future climate. Congressional reserves, which had a similar role as matrix under current climate, played as great a relative role as LSRs under future climates (Fig. 3).

\section{Performance of constrained solutions}

Similar trends in ecoprovince importance were evident for constrained solutions (Fig. 2, Fig. S3) based on localized species as were shown in unconstrained solutions (Fig. S1b). Latitudinal position, elevational position, and topographic and climatic heterogeneity remained important. However, results with solutions constrained to noncongressional reserve federal land were less stable (more sensitive to climate change) than were unconstrained results. As seen in the unconstrained solutions, patterns for owl-based solutions were generally more stable under climate change. For the owl, results from interactions scenarios were more similar to those without than was the case for localized species.

LSR, which cover $37.3 \%$ of the area considered by the constrained solutions (LSR and matrix), held a slightly higher than expected proportion of priority areas in composite constrained solutions under current $(40.2 \%$, scenario 1) and future (41.8\%; scenario 5) climate. In owl-based constrained solutions, LSR also showed a slightly greater role under both current ( $43.4 \%$; scenario $1)$ and future ( $42.4 \%$, scenario 5$)$ climate.

\section{Performance of owl as an umbrella species}

The correlation between prioritization rankings over the entire region for owl-based and localized species-based unconstrained solutions declined slightly from current (0.363; scenario 1) to future climates (0.324 and 0.301 , scenarios 2 and 4), but increased (0.433 and 0.459, scenarios 3 and 5) in the interaction scenarios where refugia (those areas with current and future habitat in proximity) were prioritized (Table S4). Lower correlation $(0.150)$ was evident in constrained solutions under current climate. The degree of correlation for constrained solutions also decreased with climate change (0.121 and 0.043, scenarios 2 and 4), but increased (0.207 and 0.172 , scenarios 3 and 5) under the interaction scenarios (Table S4). Owl-based constrained solutions captured between $37.7 \%$ and $43.9 \%$ (depending on scenario) of constrained localized-species based priority areas, not considering congressional reserves, with overlap highest for the interaction scenarios. This was similar to both the proportion expected based on area (37.3\%), and that captured by current LSR (37.2-41.7\%, depending on scenario). When additive-benefit selection was used, correlations were higher for both unconstrained (0.811) and constrained (0.343) solutions.

Owl-based solutions captured more owl habitat than solutions based on localized species' habitat, with solutions based on composite goals intermediate in performance (Fig. 4). Owl-based solutions also captured the highest mean proportion of habitat for localized species. A localized species-based solution performed poorest by this metric, with the composite solution intermediate in performance. However, a greater number of localized species were poorly represented (had a low proportion of their habitat conserved) in owl-based solutions (Fig. 4). Additionally, owl-based solutions were less successful at capturing the core areas of localized species, as measured by the greater mean proportion of the total solution required to capture $90 \%$ of the total occurrence probability for a species (Fig. S4). Solutions became on average less able to efficiently capture core areas as climate changed. Minimum values for representation of localized species in both composite and localized species-based solutions declined slightly with climate change, but remained higher than those in owl-based solutions (Fig. 4). When compared with localized species-based solutions, solutions based on composite 


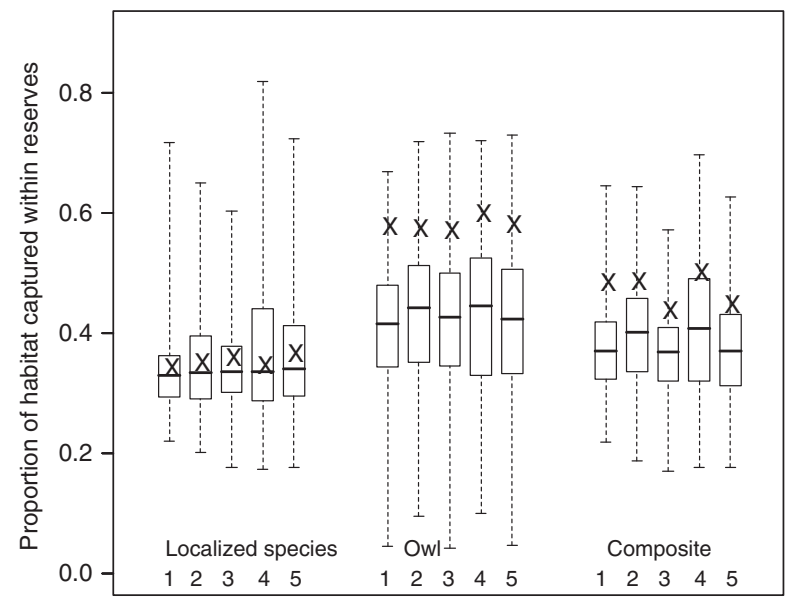

Fig. 4 Relative performance of unconstrained solutions under scenarios 1 through 5 optimized for alternate conservation objectives: localized species habitat goals, Northern Spotted Owl habitat goals, and composite goals (Fig. 2). Boxplots show the distribution (mean, $25 \%$ and $75 \%$ percentiles, and extremes) of the proportion of total regional habitat protected within the reserve system identified by ZONATION for the 130 localized species. Crosses indicate the proportion of total regional habitat remaining for the owl.

goals achieve increased owl habitat representation while retaining similar minimum representation for localized species.

\section{Discussion}

The effectiveness of a fixed system of reserves may be compromised under climate change, a problem that may be compounded when well-studied vertebrate species are used as umbrellas for other taxa (Andelman \& Fagan, 2000; Araújo et al., 2004). As species' niches shift individualistically, species whose distribution coincidentally overlaps under current climate may diverge in distribution under future climates. Our results suggest that both the US federal NWFP and more optimal reserve solutions based on current knowledge of owl habitat overlap areas of high localized-species richness but poorly capture core areas of localized species' distribution. However, congruence between priority areas across taxa is greater when refugia where current and future habitat is in proximity are prioritized. Although selection strategies that prioritize core areas of species ranges' may enhance persistence, they increase contrasts in priority areas across taxa and under climate change, necessitating explicit consideration of habitat of both focal species and lesser-known taxa under both current and future climates.

Conservation planning is by its nature an iterative process, due to increasing knowledge, changing land- scapes, and shifting societal values (Raphael \& Molina, 2007). Although it is appropriate to review the adequacy of the NWFP's management allocations, we must take as context other aspects of that planning process such as goals for maintaining geographically well-distributed populations and spreading economic impacts throughout the region. Our results are one source of qualitative and quantitative insights on regional conservation priorities in the face of changing climate that would need to be modified in a second planning phase to identify a system of reserves of sufficient size and connectivity that was most congruent with areas prioritized in our results (e.g., Fig. 1). Integrating multispecies representation and persistence (the issue of 'how much is enough?') is a challenging frontier of conservation planning (Carroll et al., 2003; Pressey et al., 2007). In this case, persistence could be assessed by population modeling which might build on initial solutions produced by ZONATION. Because we lack guidance on persistence thresholds derived from such population models, we could have plausibly evaluated solutions based on a contraction or expansion, rather than reallocation, of current reserve area. We used current reserve system extent as a minimum solution in our analysis because the extent of the region's older forest remains at historic lows and the localized species we considered are only a subset of old-forest associated species of concern (Molina et al., 2006; Noon \& Blakesley, 2006; Strittholt et al., 2006).

Because climatic refugia and other key areas can be identified at a regional scale, a broad-scale analysis such as ours is a key component in improving the efficiency and efficacy of localized species conservation through a multiscale conservation strategy focusing on both sites and larger reserves (Molina et al., 2006). For example, our results may serve to help overcome past problems in integrating the 'Survey and Manage' program with other land management goals, by offering a method to retain the NWFP's taxonomically broad conservation focus while reducing requirements for preproject surveys where a species is unlikely to occur, or not surveying and assuming presence where model predictions suggest the species is likely to occur (Dunk et al., 2004).

The climatic niche modeling approach used here, which predicts current and future species distribution based on correlations between occurrence data and climatic variables, has important limitations. Distribution of most species is influenced by a host of additional factors such as interactions with competing species or other limitations on a species' fundamental niche (Lawler et al., 2009). Climatic niche models can be justified as allowing a first approximation of potential responses to climate change when assessing large groups of poorly known species. For example, the lack of knowledge of 
more than the most basic natural history for the majority of the localized species considered here precludes development of more complex mechanistic models (Raphael \& Molina, 2007). Given these limitations, we found, as have previous studies, that the MAXENT method provides a robust means of developing niche models from nonsystematic presence-only data across a range of sample sizes (Elith et al., 2006; Hernandez et al., 2006; Pearson et al., 2007; Wisz et al., 2008).

Our suite of species generally showed a strong association with older forest. Combined climate and vegetation models were superior to climate-only models for 127 of 131 species. Although we used statistically downscaled climate variables at the highest spatial resolution available for our study region, there remained a strong contrast in initial spatial resolution between climate and vegetation variables. Because vegetation at $100 \mathrm{~m}$ resolution was important in over $85 \%$ of localized species models, we judged that resampling of both vegetation and climate data to this intermediate spatial resolution was a superior strategy to aggregating vegetation data to very coarse spatial scales $(10 \mathrm{~km})$.

Although ideally we would have projected future species distributions based on dynamic models that project vegetation response to climate, such models typically predict vegetation response at the biome level, and thus lack the spatial resolution ( $1 \mathrm{ha})$, thematic detail (forest age class), and regional geographic extent necessary for our analyses (Purves \& Pacala, 2008). In Pacific Northwest forests, stands may remain in the old forest age class (>150 years; Strittholt et al., 2006) for centuries unless harvested or subject to stand-replacing fire disturbance. Thus our assumption that old and mature forest vegetation classes remain static under the timeframe of our projections (50-100 years) allowed a useful first approximation of climate change impacts with better predictive performance than would environmental niche models based exclusively on climate (Beale et al., 2008). Future research incorporating feedback between climate change, vegetation type, and disturbance regimes would be necessary to refine this assessment (Keith et al., 2008; Purves \& Pacala, 2008).

Our results suggest that the NWFP's LSR successfully prioritized areas with greater biological importance for the owl than existing congressional reserves. We found that the owl's umbrella effect under current climate, although much better than expected by chance, was poor enough to suggest the need for explicitly considering both focal and localized species, as in our composite solutions. The contrasts evident between the owl and localized species can be attributed to contrasts in underlying climate-habitat relationships, greater dominance of vegetation variables in the owl MAXENT models, and the broader scale of habitat use and larger dispersal distance in the owl. Reserve solutions based on the owl overlap areas of high localized-species richness but poorly capture core areas of localized-species' distribution. However, the owl's effectiveness as an umbrella species is enhanced under climate change in those scenarios that prioritize refugia through consideration of interactions between current and future habitat (Table S4). In addition, finer-scale NWFP reserve allocations such as Riparian Reserves (stream buffer zones) that were not considered here enhance the capacity of the current reserve network to protect riparian-dependent localized species.

The owl's performance as an umbrella species was poorer in the constrained solutions (Fig. 2), which limited the area of evaluation to the matrix and LSR category. Both LSR and optimal owl-based priority areas performed little better than expected based on area alone at capturing localized-species based priority areas. This is because the poor value of private lands and high value of congressional reserves for both the owl and localized species creates commonalities in unconstrained solutions that are not considered when evaluating the constrained solutions. Whereas in this study we report general conclusions for the suite of localized species, the individual species models can be used to refine the NWFP's coarse- and fine-filter approach by identifying those localized species for which the owl serves as a good umbrella species, as well as species that will continue to need more individualized attention.

The general pattern in our results was intuitive, in that as climate warms, priority areas shift northward and to higher elevations. For localized species, unlike for the owl, congressional reserves, commonly considered to be dominated by biologically unimportant 'rock and ice,' show higher priority in our results than the LSR added by the NWFP. This contrast increases under climate change. The changing relative importance of management categories may be attributable to contrasts in their mean elevation $(408,973,943$, and $1360 \mathrm{~m}$ for private land, matrix, LSR, and congressional reserves, respectively). Less intuitively, our results identified retention of priority areas in cooler coastal habitat, and the continued importance under future climate of paleorefugia (Stebbins \& Major, 1965; Loarie et al., 2008). Little contrast was evident between solutions using contrasting levels of uncertainty discounting (reduction in habitat value based on variation between future habitat predictions), implying that areas with the best habitat also have low uncertainty across climate projections (C. Carroll, unpublished results).

Contrast between the performance of reserve systems based on different goals (e.g., owl vs. localized species habitat) are relatively large (Fig. 4). For example, 
solutions based on the owl result in many localized species being practically excluded from the reserve system. However, contrasts between the performance of reserve system based on a particular goal under different climates may appear low (Fig. 4). This is in part because performance is measured relative to habitat maps unique to a scenario (e.g., the current scenario's success at capturing current habitat). In addition, constrained scenarios (Figs S1b and S3) are made more similar by the limitations to reserve placement imposed by current management boundaries (Fig. 2). Priority areas optimized for multiple species would be expected to be more stable under climate change than the range of any single species. More fundamentally, however, the effectiveness of reserve systems developed by consideration of current-future habitat proximity (scenarios 3 and 5; Fig. 2) demonstrates that fixed reserve networks optimized for climate change can adequately capture both current and future habitat.

The relative importance of the different ecoprovinces remained more stable across the five scenarios for the owl than was the case for localized species. When compared with localized species, high-quality owl habitat is intermixed with poor habitat throughout a broad geographic range. This highlights inherent contrasts in the scale of potential climate change adaptation strategies for wide-ranging and localized species. Although our results project a northward movement and expansion of the owl's optimal climatic range, this result must be qualified by contemporary data showing increasing displacement of spotted owl by the congeneric barred owl (Strix varia), especially in the northern portion of its range (Noon \& Blakesley, 2006). This emphasizes the need for development of multispecies models of climate change effects.

The ZONATION landscape prioritization algorithm can identify areas based on prioritization of the core areas of each species distribution (i.e., where occurrence values are highest; the approach used in this study). Alternatively, ZONATION can develop additive-benefit solutions that prioritize areas which show maximum total occurrence over all species (Moilanen, 2007). Additivebenefit localized species solutions resembled owl-based core-area solutions (Fig. S1) in their focus on speciesrich areas within ecoprovinces at the center of the region. Core-area-based solutions for localized species additionally focus on geographically marginal ecoprovinces that hold habitat atypical of the region (Fig. S1).

Each of these contrasting approaches can appear best depending on the metric used to evaluate them. Although core-area ZONATION solutions attempt to optimally capture areas of highest habitat suitability, they may not maximize simpler metrics such as mean re- presentation. Areas of highest-quality owl habitat coincide with areas of high localized species richness, and thus coincidentally achieve high mean representation for localized species. However, they achieve lower minimum representation and poorer representation of areas of high occurrence probability. Solutions based on composite goals combine higher owl habitat representation with high minimum representation for localized species.

Both minimum representation levels and efficiency at capturing species core areas declined under future climates (Fig. 4 and S4). This may be due to the geographic divergence of communities (species ranges) with climate change and the divergent impacts of climate change on range size for different species. Although it is not well known how the demographic role of an area scales with occurrence values, core-areabased prioritization is plausibly a better method for capturing key source areas than is an additive-benefit approach that may capture large areas of moderate quality habitat for a species (Moilanen, 2007). However, the conceptual basis for when to use each of these two methods needs to be further explored by evaluating resultant solutions for species persistence using population models (Keith et al., 2008).

Our results suggest that the current reserve system will face challenges conserving its current suite of species under future climates. However, fixed reserve networks built with a consideration of climate change, as in our interaction scenarios, may be relatively effective at maintaining species due to inclusion of areas of climatic and topographic heterogeneity that allow even species with limited dispersal to colonize future habitat. The interactions scenario solutions, when compared with solutions designed around a single climate period, showed more stable habitat value, greater commonality between priority areas for different species, and resultant better performance of the owl as an umbrella species. Forest practices that increase older forests could also mitigate against adverse climate impacts by adding more patches of suitable habitat within the climate envelope of each species. Reserve selection approaches that prioritize core areas of species ranges' may enhance persistence over species-richness based approaches, but appear to increase contrasts in priority areas between taxa and between current and future habitat. Thus use of core-area selection strategies should be combined with a broadened taxonomic scope and increased attention to habitat change.

Evaluating climate-driven range shifts for species with restricted ranges poses a challenge given the typically coarse resolution of climate projections (Lawler et al., 2009), but may strengthen the effectiveness of conservation plans (Franco et al., 2009). The importance 
of vegetation variables in $97 \%$ of our species' models emphasizes the need to integrate diverse data into niche models and develop finer-resolution dynamic vegetation models (Keith et al., 2008; Purves \& Pacala, 2008). Our results suggest that, especially in regions that retain large areas of natural vegetation, systems of fixed reserves can be designed to be more resilient to climate change, thus greatly improving the chances of retaining a large component of the biological diversity of forest ecosystems.

\section{Acknowledgements}

We thank the US Forest Service, Bureau of Land Management, and California Department of Fish and Game for providing owl or localized species survey data, and E. Salathé for providing future climate projections. M. Visser, S. Phillips, H. Kujala, and two anonymous reviewers provided helpful comments. A. M. thanks the Academy of Finland centre of excellence program 2006-2011 for support.

\section{References}

Andelman SJ, Fagan WF (2000) Umbrellas and flagships: efficient conservation surrogates or expensive mistakes? Proceedings National Academy of Sciences USA, 97, 5954-5959.

Araújo MB, Cabeza M, Thuiller W, Hannah L, Williams PH (2004) Would climate change drive species out of reserves? An assessment of existing reserve selection methods. Global Change Biology, 10, 1618-1626.

Araújo MB, New M (2007) Ensemble forecasting of species distributions. Trends in Ecology and Evolution, 22, 42-47.

Beale CM, Lennon JJ, Gimona A (2008) Opening the climate envelope reveals no macroscale associations with climate in European birds. Proceedings National Academy of Sciences USA, 105, 14908-14912.

Ben-Haim Y (2006) Information-Gap Decision Theory: Decisions Under Severe Uncertainty, 2nd edn. Academic Press, London, UK.

Carroll C, Johnson DS (2008) The importance of being spatial (and reserved): assessing Northern Spotted Owl habitat relationships with hierarchical Bayesian models. Conservation Biology, 22, 1026-1036.

Carroll C, Noss RF, Paquet PC, Schumaker NH (2003) Use of population viability analysis and reserve selection algorithms in regional conservation plans. Ecological Applications, 13, 1773-1789.

Dunk JR, Zielinski WJ, Preisler HK (2004) Predicting the occurrence of rare mollusks in northern California forests. Ecological Applications, 14, 713-729.

Elith J, Graham CH, Anderson RP et al. (2006) Novel methods improve prediction of species' distributions from occurrence data. Ecography, 29, 129-151.

Franco AMA, Anderson BJ, Roy DB, Gillings S, Fox R, Moilanen A, Thomas CD (2009) Surrogacy and persistence in reserve selection: landscape prioritization for multiple taxa in Britain. Journal of Applied Ecology, 46, 82-91.
Goodchild MF (1986) Spatial Autocorrelation. Geo Books, Norwich, UK.

Hernandez PA, Graham CH, Master LL, Albert DL (2006) The effect of sample size and species characteristics on performance of different species distribution modeling methods. Ecography, 29, 773-785.

Keith DA, Akçakaya HR, Thuiller W et al. (2008) Predicting extinction risks under climate change: coupling stochastic population models with dynamic bioclimatic habitat models. Biological Letters, 4, 560-563.

Kremen C, Cameron A, Moilanen A et al. (2008) Aligning conservation priorities across taxa in Madagascar, a biodiversity hotspot, with high-resolution planning tools. Science, 320, 222-226.

Lawler JJ, Shafer SL, White D, Kareiva P, Maurer EP, Blaustein AR, Bartlein PJ (2009) Projected climate-induced faunal change in the Western Hemisphere. Ecology, 90, 588-597.

Lawler JJ, White DS, Neilson RP, Blaustein AR (2006) Predicting climate-induced range shifts: model differences and model reliability. Global Change Biology, 12, 1568-1584.

Leathwick JR, Moilanen A, Francis M, Elith J, Taylor P, Julian K, Hastie T (2008) Novel methods for the design and evaluation of marine protected areas in offshore waters. Conservation Letters, 1, 91-102.

Loarie SR, Carter BE, Hayhoe K, McMahon S, Moe R, Knight CA, Ackerly DD (2008) Climate change and the future of California's endemic flora. PLoS ONE, 3, e2502, 1-10.

Maurer EP, Wood AW, Adam JC, Lettenmaier DP, Nijssen B (2002) A long-term hydrologically based data set of land surface fluxes and states for the conterminous United States. Journal of Climate, 15, 3237-3251.

Moilanen A (2007) Landscape zonation, benefit functions and target-based planning: unifying reserve selection strategies. Biological Conservation, 134, 571-579.

Moilanen A, Franco AMA, Early R, Fox R, Wintle B, Thomas CD (2005) Prioritising multiple-use landscapes for conservation: methods for large multi-species planning problems. Proceedings Royal Society of London, Series B, 272, 1885-1891.

Moilanen A, Kujala H (2008) Zonation: software for spatial conservation prioritization. User Manual v2.0. Metapopulation Research Group, University of Helsinki, FI. Available at http://www.helsinki.fi/bioscience/consplan/software/Zonation/index.html (accessed November 2008).

Moilanen A, Kujala H, Leathwick J (2009) The zonation framework and software for conservation prioritization. In: Spatial Conservation Prioritization: Quantitative Methods and Computational Tools (eds Moilanen A, Wilson KA, Possingham HP), Oxford University Press, Oxford, UK.

Moilanen A, Wintle BA, Elith J, Burgman M (2006) Uncertainty analysis for regional-scale reserve selection. Conservation Biology, 20, 1688-1697.

Molina R, Marcot BG, Lesher R (2006) Protecting rare, oldgrowth, forest-associated species under the survey and manage program guidelines of the northwest forest plan. Conservation Biology, 20, 306-318.

Noon BR, Blakesley JA (2006) Conservation of the Northern Spotted Owl under the Northwest Forest Plan. Conservation Biology, 20, 288-296. 
Noss RF, Cooperrider A (1994) Saving Nature's Legacy: Protecting and Restoring Biodiversity. Island Press, Washington, DC.

Olson DH, Van Norman KJ, Huff RD (2007) The utility of strategic surveys for rare and little-known species under the Northwest Forest Plan. General Technical Reports PNW-GTR-708, USDA Forest Service Pacific Northwest Research Station, Portland, OR.

Pearson RG, Raxworthy CJ, Nakamura M, Peterson AT. (2007) Predicting species' distributions from small numbers of occurrence records: a test case using cryptic geckos in Madagascar. Journal of Biogeography, 34, 102-117.

Phillips SJ, Anderson RP, Schapire RE (2006) Maximum entropy modeling of species geographic distributions. Ecological Modelling, 190, 231-259.

Phillips SJ, Dudik M (2008) Modeling of species distributions with Maxent: new extensions and a comprehensive evaluation. Ecography, 31, 161-175.

Phillips SJ, Dudik M, Elith J, Graham CH, Lehmann A, Leathwick J, Ferrier S (2009) Sample selection bias and presenceonly distribution models: implications for background and pseudo-absence data. Ecological Applications, 19, 181-197.

Pressey RL, Cabeza M, Watts ME, Cowling RM, Wilson KA (2007) Conservation planning in a changing world. Trends in Ecology and Evolution, 22, 583-592.

Purves D, Pacala S (2008) Predictive models of forest dynamics. Science, 320, 1452-1453.

Rahmstorf S, Cazenave A, Church J, Hansen J, Keeling R, Parker D, Somervillle RCJ (2007) Recent climate observations compared to projections. Science, 316, 709.

Raphael MG, Molina R (2007) Conservation of Rare or Little-Known Species: Biological, Social, and Economic Considerations. Island Press, Washington, DC.
Rayfield B, Moilanen A, Fortin M-J (2009) Incorporating consumer-resource spatial interactions in reserve design. Ecological Modelling, 220, 725-733.

Salathé EP (2005) Methods for selecting and downscaling simulations of future global climate with application to hydrologic modeling. International Journal of Climatology, 25, 419-436.

Stebbins GL, Major J (1965) Endemism and speciation in the California flora. Ecological Monographs, 35, 2-35.

Strittholt JR, Dellasala DA, Jiang H (2006) Status of mature and old-growth forests in the Pacific Northwest. Conservation Biology, 20, 363-374.

Thomas JW, Franklin JF, Gordon J, Johnson KN (2006) The Northwest Forest Plan: origins, components, implementation experience, and suggestions for change. Conservation Biology, 20, 277-287.

Thomson J, Moilanen A, Vesk P, Bennett AF, McNally R (2009) Where and when to revegetate: a quantitative method for scheduling landscape reconstruction Ecological Applications, in press.

US Department of Agriculture Forest Service, US Department of Interior Bureau of Land Management (1994) Final supplemental environmental impact statement on management of habitat for latesuccessional and old-growth forest within the range of the Northern Spotted Owl, USDA Forest Service Portland, Oregon.

VanDerWal J, Shoo LP, Graham C, Williams SE (2009) Selecting pseudo-absence data for presence-only distribution modeling: how far should you stray from what you know? Ecological Modelling, 220, 589-594.

Wisz MS, Hijmans RJ, Li J, Peterson AT, Graham CH, Guisan A (2008) Effects of sample size on the performance of species distribution models. Diversity and Distributions, 14, 763-773. 


\section{Supporting Information}

Additional Supporting Information may be found in the online version of this article:

Table S1. Localized species considered in Zonation reserve selection analysis for the Pacific Northwest. Species with less than 10 locations after thinning of locations to $>1 \mathrm{~km}$ separation were excluded from further analysis.

Table S2. List of candidate models compared in Maxent modeling of old-growth associated species in the Pacific Northwest.

Table S3. Patterns of change in the proportion of each ecoprovince within priority areas for old-growth associated species in the Pacific Northwest as identified by the Zonation solutions. Ecoprovince abbreviations are as used in Fig. 1.

Table S4. Correlations between priority rankings produced by Zonation runs based on localized species and on Northern Spotted Owl habitat in the Pacific Northwest. Unconstrained solutions identified priority areas irrespective of management category, whereas constrained solutions could reallocate reserve status within the matrix and LSR categories only.

Figure S1. Proportion of each ecoprovince within the Zonation solution (network of priority areas) under a) unconstrained, and b) constrained composite-goal scenarios 1 (current habitat), 2 (near-future habitat), 3 (interacting current and near-future habitat), 4 (distant future habitat), and 5 (interacting current and distant future habitat). Constrained Zonation solutions could reallocate reserve status within the matrix and LSR categories only whereas unconstrained solutions allocated reserve areas irrespective of existing management category.

Figure S2. Distribution of priority areas identified by Zonation for conservation of the Northern Spotted Owl and 130 localized species under unconstrained scenarios 1 (current habitat), 2 (near-future habitat), 3 (interacting current and near-future habitat), 4 (distant future habitat), and 5 (interacting current and distant future habitat). Unconstrained Zonation solutions could reallocate reserve status irrespective of existing management category. Area of the reserve network was set equal to that of the current system of congressional reserves and late-successional reserves ( $\sim 28 \%$ of analysis area).

Figure S3. Distribution of priority areas identified by Zonation for conservation of the Northern Spotted Owl and 130 localized species under constrained scenarios 1 (current habitat), 2 (near-future habitat), 3 (interacting current and near-future habitat), 4 (distant future habitat), and 5 (interacting current and distant future habitat). Constrained Zonation solutions could reallocate reserves equal in area to the extent of LSR (13.9\% of analysis area) within the matrix and LSR categories only. Total area of the reserve network, as in Fig. S3, was set equal to that of the current system of congressional reserves and late-successional reserves ( $\sim 28 \%$ of analysis area).

Figure S4. The mean proportion of the Zonation solution required to capture $90 \%$ of the predicted occurrence value of a species contained within the entire Zonation solution. The average was taken over all 131 species considered. Results from three scenarios (scenario 1 (current), scenario 2 (near-future), scenario 4 (distant future), and scenario 1 using an additive-benefit selection function) are shown under three contrasting Zonation goals sets: localized species only, owl only, and composite goals. A solution that requires a larger proportion of the total solution to encompass $90 \%$ of the total value per species is opting against core areas of high occurrence value for particular species in favor of larger areas of moderate value for multiple species.

Please note: Wiley-Blackwell are not responsible for the content or functionality of any supporting materials supplied by the authors. Any queries (other than missing material) should be directed to the corresponding author for the article. 